\title{
Knee Osteoarthritis Treated with Percutaneous Chondral-Bone Interface Optimization: A Pilot Trial
}

\author{
Vijay Vad1, Raghav Barve' ${ }^{2}$, Erica Linnell ${ }^{*}$, Julian Harrison'1 \\ ${ }^{1}$ Hospital for Special Surgery, New York, NY, USA \\ ${ }^{2}$ Ruby Hall Clinic, Pune, India \\ Email: *erica.j.linnell@gmail.com
}

Received 2 November 2015; accepted 5 January 2016; published 8 January 2016

Copyright (C) 2016 by authors and Scientific Research Publishing Inc.

This work is licensed under the Creative Commons Attribution International License (CC BY). http://creativecommons.org/licenses/by/4.0/

\section{c) (i) Open Access}

\begin{abstract}
Objective: The objective is to evaluate the efficacy of using tibial bone marrow delivered to the chondral-bone interface (CBI) via percutaneous chondral bone interface optimization (PeCaBoo) as a therapy for knee osteoarthritis (OA). Study Design: A series of prospective cases were presented. Participants: Our study included 10 patients with medial or lateral compartment knee $0 A$. Methods: With 1 cc of heparin pre-loaded in the syringe, 5 cc of tibial bone marrow was withdrawn from the proximal tibia. The resultant $6 \mathrm{cc}$ of aspirate in the syringe was injected via PeCaBoo, 2 cc at a time, into the superior $\mathrm{CBI}$ and inferior $\mathrm{CBI}$. The remaining 2 cc was injected via needle into the intra-articular joint space. Main Outcome Measurements: Patients had MRIs taken pre-procedure and 3 months post-procedure to measure bone edema and intra-articular matrix thickness. Patient-reported outcomes recorded included the Western Ontario and McMaster University Osteoarthritis Index (WOMAC) and the Numeric Paint Rating Scale (NRS), which were both obtained pre-procedure and post-procedure at 3, 6, and 12 months. Use of non-steroidal anti-inflammatories (NSAIDs) was recorded pre- and post-procedure as well. Results: Our study included 4 males and 6 females, with an average age of 63.5 years. The average follow-up time was 14 months, with a range of 13 - 15 months. The mean WOMAC score was 58.2 points pre-procedure and 35.3 points post-procedure $(p<0.01)$. The mean NRS-Pain score was 8.6 points pre-procedure and 2.8 points post-procedure $(p<0.01)$. The matrix thickness increased by $14 \%$ on average at 3 months postprocedure $(p<0.01)$. The proportion of patients taking NSAIDs decreased by $60 \%$ after the PeCaBoo procedure. The subgroup of patients with tibial edema and knee $\mathrm{OA}$ had optimal outcomes. Conclusions: Tibial bone marrow stem cell delivered via PeCaBoo is a novel minimally-invasive treatment for knee $0 A$, with potential to repair cartilage and improve knee pain and function.
\end{abstract}

${ }^{*}$ Corresponding author. 
Keywords

Osteoarthritis, Stem Cell, Allografts, Chondral-Bone Interface

\section{Introduction}

Knee osteoarthritis (OA) is a leading cause of disability, affecting more than 20 million people in the United States and over 266 million worldwide [1] [2]. The economic cost of OA is substantial; it is one of the leading causes of hospitalizations and the primary indicator for joint replacement surgery [3]. In 2009, nearly one million knee and hip replacements amounted to $\$ 42.3$ billion in costs [3]. Joint replacement surgery can also have major complications, such as deep venous thrombosis and neurovascular complications [4]. This has led to a search for new minimally-invasive, lower cost techniques for the long-term treatment of OA with fewer complications [5] [6].

Studies have indicated that vascular pathology plays a major role in the degeneration of the chondral-bone interface (CBI) in OA [7]-[9]. Ischemia promotes catabolic processes and production of pro-inflammatory factors in chondrocytes, predisposing cartilage to degeneration [8]. Decreased nutrient and gas exchange due to subchondral ischemia stimulates apoptosis of osteocytes, leading to bone resorption by osteoclasts and decreased support for overlying cartilage [7]. Significant ischemia is also associated with bone marrow edema-like lesions seen on magnetic resonance imaging (MRI) of the knee [10] [11].

An innovative therapy for the treatment of knee OA is a percutaneous injection of cultured bone marrow stem cells into the affected knee joint [9]. Studies report improved functional status and patient pain scores [12], along with cartilage regeneration as seen on an MRI scans [13]. Patients with bone marrow edema and avascular necrosis have reported positive outcomes with bone marrow-derived cellular therapy as well [14].

Various bone marrow constituents can repair cartilage and restore vascularity when injected into the knee joint, including platelet-derived growth factor (PDGF), vascular endothelial growth factor (VEGF), transforming growth factor (TGF), bone morphogenetic protein (BMP), and stem cells [15] [16]. PDGF enhances angiogenesis, promotes the synthesis of collagen, and acts as a chemoattractant for mesenchymal progenitor cells [17] [18]. VEGF, a subfamily of PDGF, promotes migration and mitosis of endothelial cells in angiogenesis [19] [20]. TGF- $\beta$ promotes synthesis of collagen, fibronectin and osteonectin, which leads to deposition of bone matrix [16] [21]. When injected into long bones, TGF- $\beta$ stimulates the production of cartilage that progresses to bone through endochondral ossification [22]. BMP stimulates chondrocyte proliferation and hypertrophy [23]. Bone marrow stem cells include premature mesenchymal cells and hematopoietic cells, which together promote angiogenesis, vasculogenesis, osteogenesis, and extracellular matrix synthesis [16].

The percutaneous cartilage-bone interface optimization system (PeCaBoo) (Vad Scientific LLC, New York, $\mathrm{NY}$ ) can be used to drill a channel into the CBI for the injection of therapeutic agents. This is a minimally invasive procedure that has the additional advantage of potentially stimulating the subchondral bone marrow to produce mesenchymal stem cells (MSCs). Studies have shown that the MSCs produced via drilling into the CBI can potentially restore cartilage to the knee joint [24]-[26].

In this study, we present a novel approach for the treatment of knee OA that uses autologous tibial bone marrow MSCs injected into the knee CBI via PeCaBoo delivery. Based on the potential of this procedure to promote healing by increasing vascularity in the CBI, our hypothesis was two-fold: (1) we predicted patients to report a reduction in pain and enhancement of mobility, and (2) we predicted an increase in matrix thickness seen on MRI scans. Our study used aspiration of proximal tibia bone marrow stem cells for ease of extraction and procedure preparation, as opposed to aspiration from the iliac crest.

\section{Materials and Methods}

The purpose of this prospective, observational study was to assess the effectiveness of MSC therapy in the treatment of knee OA. We obtained approval by the institution's ethics committee and informed consent from all participants prior to conducting this study. Inclusion criteria included (1) medial or lateral compartment knee OA as defined by the Kellgren-Lawrence grading scale and (2) 6 months of failed physical therapy, oral medications, cortisone injections, hyaluronic acid injections, and arthroscopic debridement. Patients were excluded if they had a 
prior history of infection.

This study took place at the Ruby Hall Clinic in Pune, India, in 2013. Ten patients ranging from $52-73$ years of age (mean and median age of 63.5) were enrolled in the study after meeting the aforementioned criteria. The cohort was comprised of 4 males and 6 females, of which 8 were of Indian ethnicity, 1 Dutch, and 1 Caucasian. Study participants were instructed not to take nonsteroidal anti-inflammatory drugs (NSAIDs) for three days prior to the procedure and five days post-procedure. The procedure was performed under IV sedation, with IV antibiotics given during the procedure.

Prior to aspiration of MSCs, $1 \mathrm{cc}$ of heparin was added to the syringe as an anticoagulant. The same syringe was then used to aspirate $5 \mathrm{cc}$ in volume of bone marrow from the proximal tibial tubercle using the PeCaBoo system. The resulting 6 cc of volume in the syringe was split into three equal parts of 2 cc each. Using fluoroscopic guidance to ensure precise locations of injection, the PeCaBoo delivery system was used to administer 2 cc injections to the superior CBI and inferior CBI, either medially or laterally depending on the location of knee OA (Figure 1). Drilling into the CBI stimulates the MSCs in the bone marrow of the CBI to proliferate and restore intra-articular matrix, in a combined effect with the injected tibial bone marrow stem cells (Figure 2). An additional 2 cc was injected into the intra-articular knee joint space using a 22 gauge $1 \frac{1 / 2}{2}$ needle. Two days after the procedure, patients were instructed to resume walking.

All study participants had an MRI scan taken pre-procedure and another taken three months post-procedure, which was used to measure changes in intra-articular matrix thickness. Participant-reported Numeric Pain Rating Scale (NRS) [27], Western Ontario and McMaster Universities Arthritis Index (WOMAC) [28], and nonsteroidal anti-inflammatory usage was obtained pre-operatively and then at a minimum of 12 months post-operatively.

\subsection{PeCaBoo Delivery System}

The PeCaBoo system uses a specialized needle inserted through a drill bit to aspirate bone marrow from the tibia, and then deliver it to the injection sites of the CBI. In the beginning of the procedure, the needle is on the end

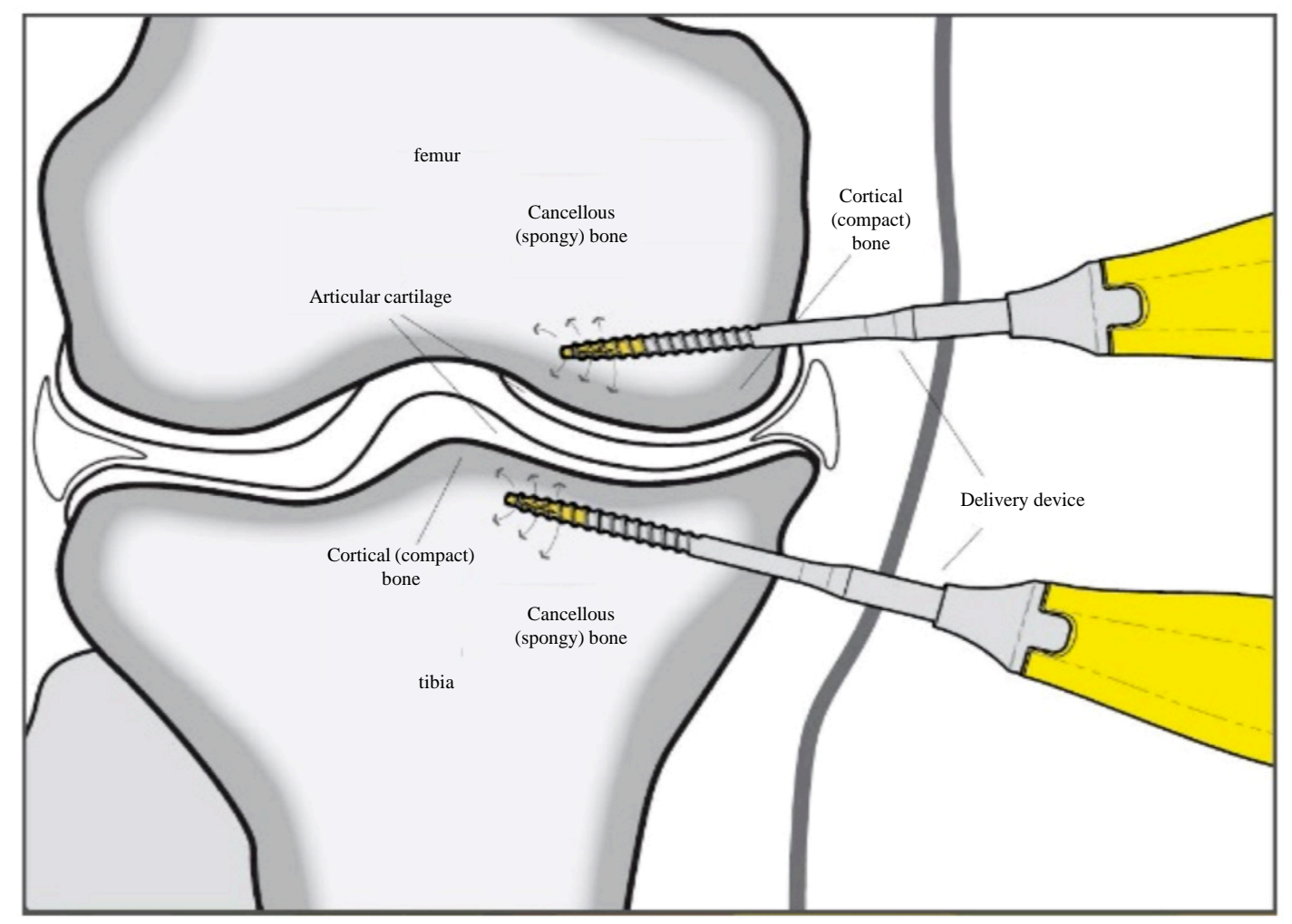

Figure 1. PeCaBoo is applied to the superior and inferior chondral-bone interfaces to stimulate the differentiation of mesenchymal stem cells into cartilage from both angles. 


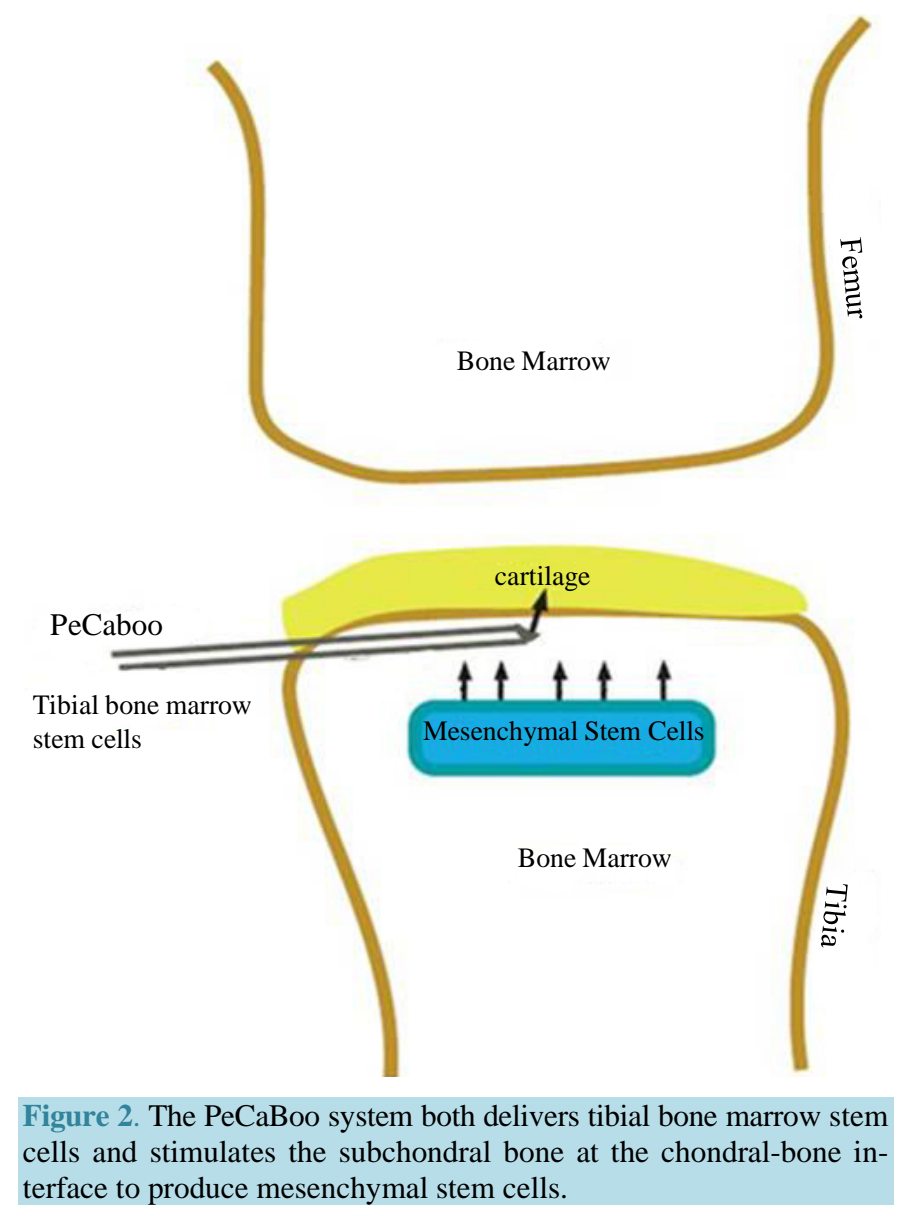

distal to the handle and is surrounded by a tapered helix that is followed by a straight helix. The tri-lobe handle contains a delivery compartment that is used to store the solution for injection. This delivery compartment can also be removed to allow leverage for inserting the needle through the hard bone superficial to the CBI. Advantages of the minimally invasive PeCaBoo delivery system include a short procedural time of several minutes to minimize trauma, air exposure, and risk of infection (Figure 3, Figure 4). The PeCaBoo delivery system was supplied by Vad Scientific LLC.

\subsection{MRI Analysis}

MRI sequences were used to measure matrix size and subchondral bone marrow edema pre-and post-operatively. Matrix increase was measured in millimeters using the sagittal view at the point of greatest thickness between the tibia and femur. The MRIs were evaluated by an independent blinded board certified radiologist.

\subsection{Statistical Analysis}

An independent blinded nurse collected the data. The data was analyzed using a paired t-test to calculate the significance between pre-and post-procedure NRS and WOMAC pain scores, and to assess their correlation with subjects' age, knee OA grade, and edema presence or absence. Alpha was set at 0.05.

\section{Results}

The cohort was comprised of 4 males and 6 females, with an average age of 63.5 years. The average patient follow-up time was 14 months, ranging from 13 - 15 months. Patient reported WOMAC score improved by 22.9 points from an average of 58.2 points pre-procedure, to 35.3 points average post-procedure $(\mathrm{p}<0.01)$ (Figure 5$)$. The NRS reported by patients also significantly improved by 5.8 points from an average of 8.6 points pre-procedure, 


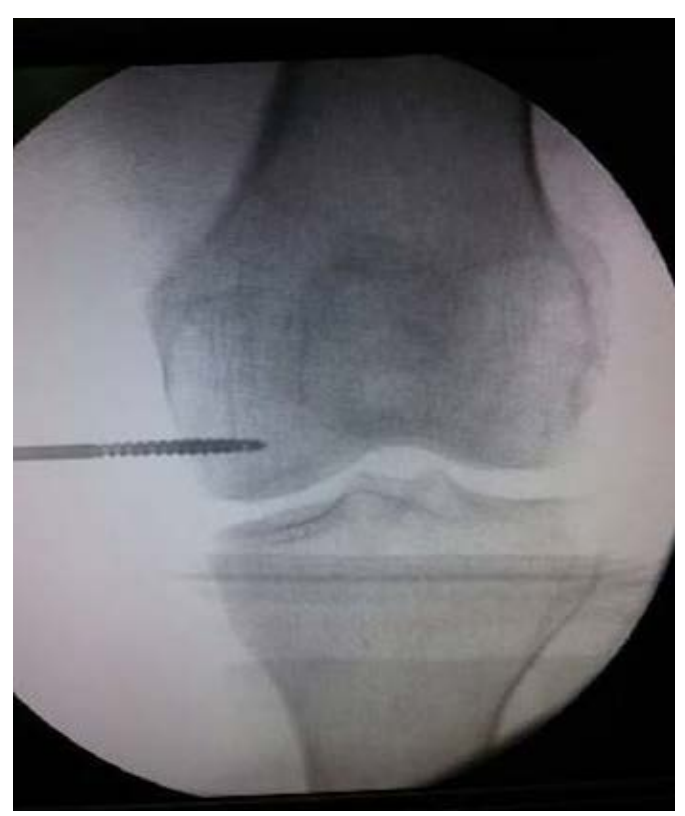

Figure 3. The PeCaBoo delivery system is positioned at the chondral-bone interface to enhance the ability of bone marrow mesenchymal stem cells to differentiate into cartilage.

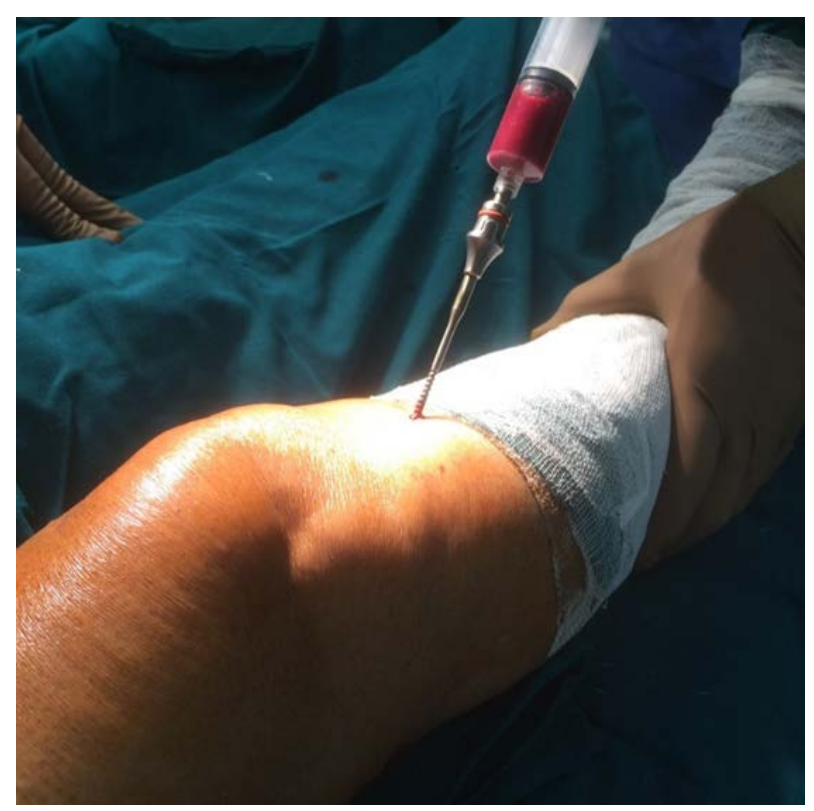

Figure 4. The minimally invasive procedure is performed under fluoroscopic guidance and intravenous sedation. The handle of $\mathrm{PeCaBoo}$ can be detached to inject therapeutic agents directly without removal of the drill.

to an average of 2.8 points post-procedure $(\mathrm{p}<0.01)$ (Figure 6).

All 10 patients were using NSAIDs pre-procedure. Only 4 patients were using NSAIDs post-procedure, resulting in a $60 \%$ decrease in study subjects who required NSAIDs to manage their knee pain.

MRI scans were used to measure the change in intra-articular matrix thickness (Figure 7). Matrix thickness increased by an average of $14.1 \%$. Four patients (40\%) did not show any restoration of matrix thickness. The six patients $(60 \%)$ who did show a restoration had an average increase of $23.5 \%$ in matrix. 


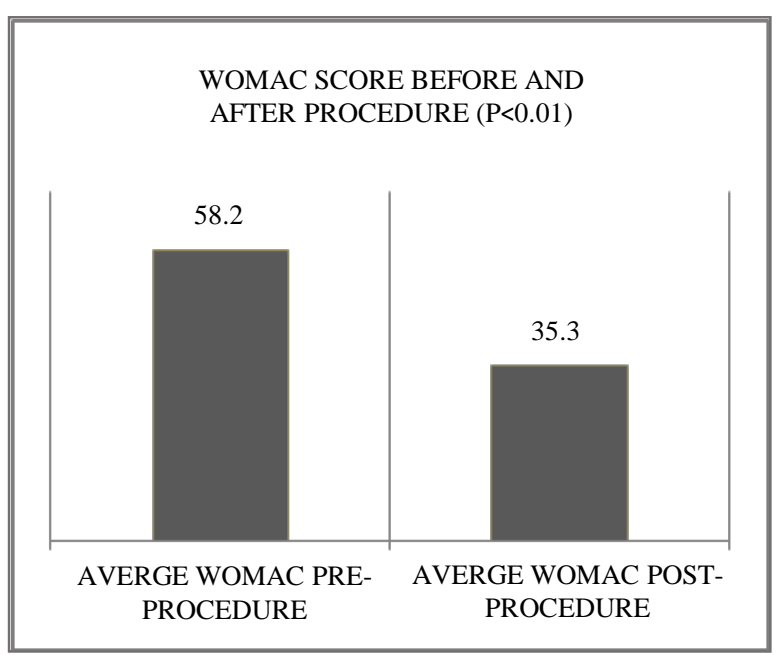

Figure 5. Data analysis found a statistically significant decrease in WOMAC score, measuring quality of life, recorded before and after the procedure at an average follow up of 14 months.

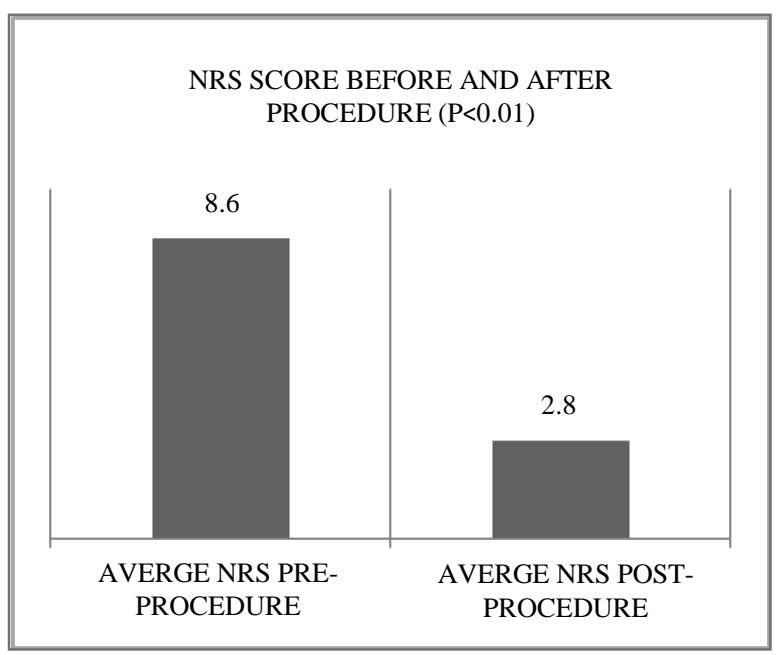

Figure 6. Data analysis found a statistically significant decrease in NRS pain score, measured on a scale from 1 to 10 , recorded before and after the procedure at an average follow up of 14 months.

Study participants ranged from 52 - 73 years of age. Both the mean and median age were 63.5 years. When the data is analyzed in a subset with those above the median age and subset below the median age, results vary between the two groups. The average NRS score improved by 7.4 points in patients below age 63.5 years, while it improved by 4.2 points in those above the median age ( $<0.01)$. Likewise, the average WOMAC score improved by 28.8 points in patients below age 63.5 years, while it improved by 17.0 points in those above the median age ( $<0.01)$. Matrix increased by an average of $16.8 \%$ in patients below age 63.5 years, with four out of five $(80 \%)$ subjects showing an increase $(\mathrm{p}<0.01)$. Participants above older than 63.5 years experienced an average increase of $11.4 \%$ in matrix, with two out of five $(40 \%)$ patients showing an increase in matrix $(\mathrm{p}<0.01)$ (Figure 8).

Data analysis by subsets of arthritis severity, as determined by the Kellgren-Lawrence grading scale, showed variation in group outcomes. The average NRS score improved by 7.2 points in patients with Grade 3 OA, while it improved by 3.8 points in patients with Grade 4 OA $(\mathrm{p}<0.01)$. Patients with Grade 3 OA had an average change 

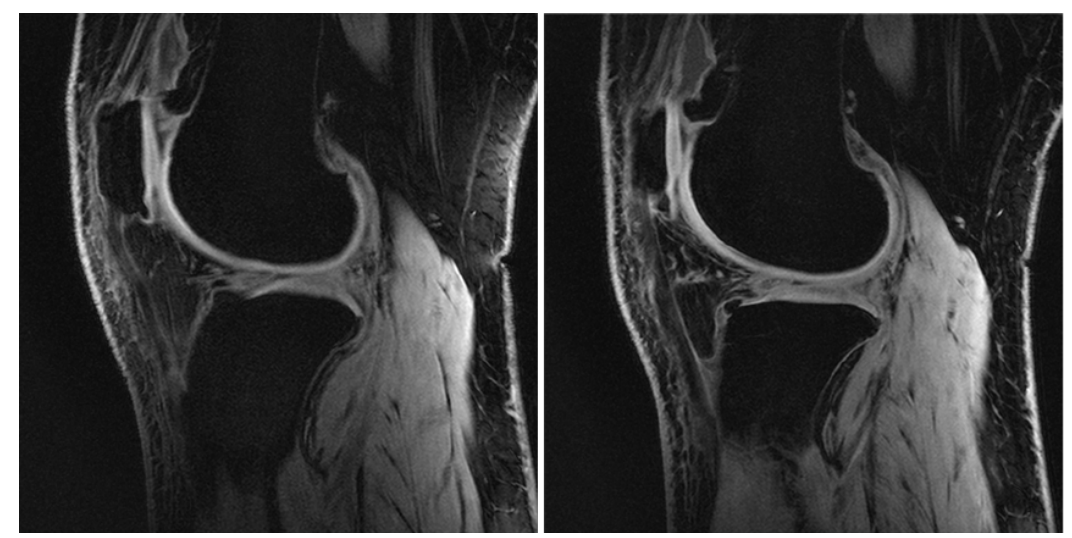

Figure 7. The pre-procedure (left) and 3 months post-procedure (right) MRI scans show an increase in intra-articular matrix. These measurements were used to determine the percentage of change in matrix thickness for each patient.

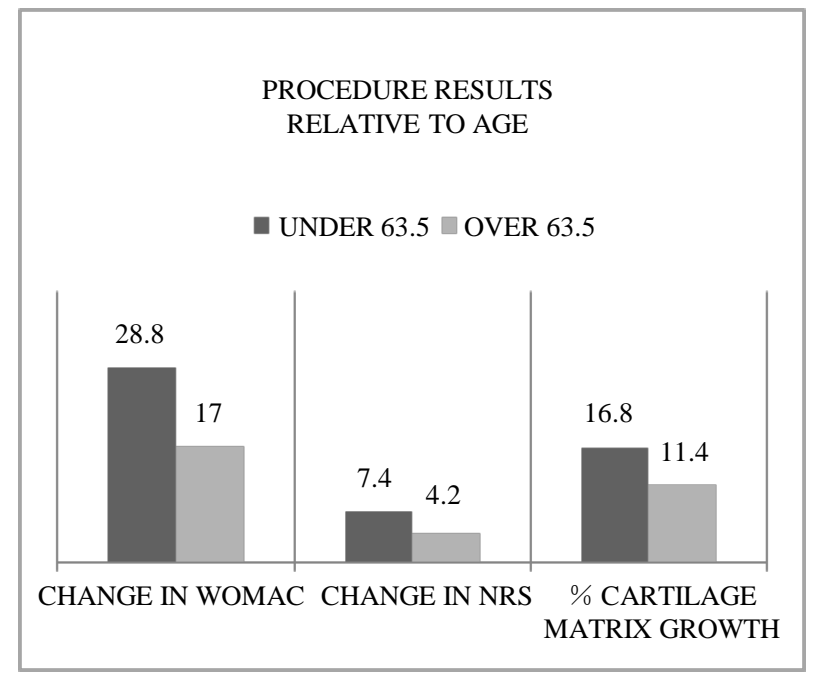

Figure 8. The procedure resulted in more positive outcomes for younger patients below the median age of 63.5 .

in WOMAC score of 27.8 points, whereas patients with Grade 4 OA had an average change of 15.5 points ( $\mathrm{p}<$ 0.01 ). The average increase in intra-articular matrix in patients with Grade 3 OA was $20.2 \%$, with five out of six (83\%) patients showing an increase $(\mathrm{p}<0.01)$. Grade 4 OA patients had an average increase in intra-articular matrix of $5 \%$, with one out of four $(25 \%)$ of patients showing an increase $(p<0.01)$. The average age of study patients with Grade 3 OA was 64 years, and the average age of patients with Grade 4 OA was 62.8 years (Figure 9).

Four patients (40\%) were found to have tibial subchondral bone marrow edema on the MRI scans. All four reported significant improvements in NRS, from an average of 9 points pre-operatively to 1.8 points post-operatively. WOMAC scores improved from 57.3 points pre-operatively to 29.5 points post-operatively. The average increase in matrix measured on the MRIs was $17.3 \%$, with three out of four of these patients showing an increase. None of them were using NSAIDs post-procedure.

Two patients (20\%) did not show significant improvements in pain scores and matrix thickness after the procedure, but they did not worsen either. Both of these patients had Grade 4 OA and were ages 68 and 73, above the median age in this study.

\section{Discussion}

Our study presents a novel approach to the treatment of knee OA that has the potential to repair cartilage and al 


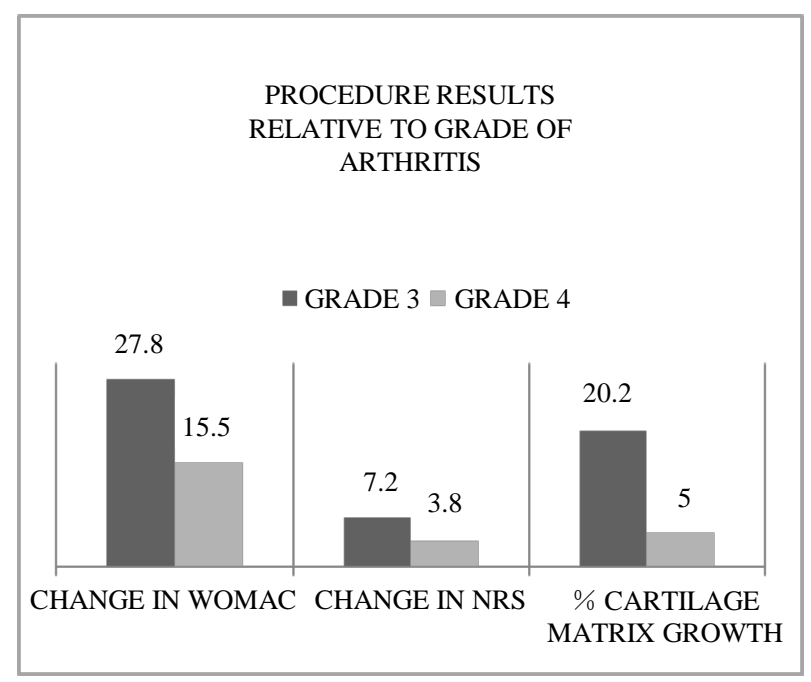

Figure 9. The procedure resulted in more positive outcomes for patients with a lower grade of arthritis. A higher grade of arthritis indicates a more severe and advanced presentation.

leviate pain over a long-term follow-up time. Using the PeCaBoo system to deliver tibial marrow cells via drilling into the CBI, our procedure is believed to stimulate angiogenesis to the subchondral bone and thereby alleviate the ischemia potentially associated with pain in knee OA. It is also thought that the injected tibial marrow mesenchymal cells can differentiate into chondrocytes and proliferate, thus increasing the intra-articular matrix size measured on MRI scans. Our results indicate that using the PeCaBoo delivery system to deliver tibial marrow cells to the CBI is a safe and effective, minimally invasive approach that could be used as an alternative treatment for patients with knee OA.

Current guidelines for the treatment of knee OA recommend the following order of therapies: (1) lifestyle modifications; (2) administer a simple analgesic (e.g. acetaminophen); (3) administer nonsteroidal anti-inflammatory drugs (NSAIDs) (e.g. COX-2 inhibitors); (4) inject hyaluronic acid or corticosteroid into the intraarticular joint space; (5) total knee replacement [2]. However, these treatments fail to effectively alleviate symptoms for many patients [2]. Many pharmaceutical companies have also recently closed research programs for OA due to the complexity of treatments and the difficulty in demonstrating their efficacy in clinical trials [29]. Thus, there exists a need for the development of new, more effective therapies to treat knee OA.

Studies have found varied results as to the efficacy of current treatments for knee OA. A 2015 study by Van der Weegen et al. found no difference between intra-articular injection of hyaluronic acid and placebo in the treatment of mild to moderate knee OA [30]. While other studies have found that hyaluronic acid injections do alleviate some pain [31], patients do not always respond to the treatment, including those enrolled in our study. Both hyaluronic acid and corticosteroid injections only have potential to provide short-term relief as well [32]. A recent meta-analysis study found NSAIDs to have a similar efficacy to hyaluronic acid in treatment of symptomatic knee OA, though long-term use of NSAIDs can result in gastrointestinal and cardiovascular complications [33] [34].

If minimally-invasive treatments fail, the common practice is to recommend total knee arthroplasty (TKA). However, this surgery has the risk of many complications including deep venous thrombosis, pulmonary embolism, and infection [35] [36]. Periprosthetic joint infection occurs in $2 \%$ of TKAs performed [37]. Infection can result in mortality, morbidity, and hospital re-admission costs that average \$20,001 per patient [38] [39]. Other possible morbidities following TKA include stiffness requiring manipulation under anesthesia, postoperative blood transfusion, and the need for reoperation [4]. The economic burden of TKAs is significant, with an average surgery cost of $\$ 24,435$ per patient that amounts to a total hospital charge of nearly $\$ 16$ billion for TKAs done nationally [40].

We hypothesize that knee OA originates at the chondral-bone interface (CBI), and therefore our procedure targets this area. When patients experience increased pressure and loss of cartilage at the CBI, the resultant ischemia shifts chondrocytes to a catabolic state and stimulates production of pro-inflammatory molecules (e.g. IL-1, 
TNF, NF-кB) and proteolytic enzymes (e.g. matrix metalloproteinases and aggrecanases) [7] [8] [41] [42]. These factors reduce synthesis and accelerate breakdown of the cartilage matrix [43].

When the mesenchymal stem cells (MSCs) from the tibial bone marrow are injected into the CBI, they have a trophic effect on chondrocytes [43]. These factors can suppress the local immune system, inhibit fibrosis and apoptosis, stimulate angiogenesis, and promote mitosis of tissue-intrinsic stem or progenitor cells [44]. MSCs are also multilineage progenitors that can differentiate into chondrocytes and thus help to restore cartilage matrix volume [45]. This is supported by our data, which shows an increase in intra-articular matrix size on MRI scans and a significant reduction in OA symptoms on the WOMAC and NRS scales after injection with MSCs.

A recent study estimates the mononucleated cell (MNC) concentration of the tibia to be $1.7 \mathrm{million} / \mathrm{ml}$, and these cells have markers highly specific to MSCs (e.g. CD 105+, CD 90+, CD 73+, CD 34-, CD 45-) [15]. When cultured in specific media, these MSCs sufficiently differentiate into chondrocytes, osteoblasts, and adipocytes [15]. We thus estimate that 8.5 million MNCs are contained within the 5 cc of aspirate withdrawn from the tibial bone marrow in our study, and that the MSCs differentiate into chondrocytes and osteoblasts to restore the CBI.

Aging is associated with a decrease in number of bone marrow MSCs, along with an impaired proliferation and chondrogenic response [46]. This is supported by our finding that younger age is associated with greater improvement in pain scores and increases in intra-articular matrix. Similarly, a lower grading of OA severity is associated with improved outcomes after the procedure.

Chronic ischemic bone disease can lead to subchondral bone marrow edema [47]. Both tibial marrow MSCs and endothelial cells express VEGF and CD105, indicating that these MSCs may contribute to angiogenesis [15] [48]. It is also known that bioactive factors secreted by MSCs can stimulate angiogenesis [44]. We believe that the MSCs used in our procedure restore vascularity to the CBI, as indicated by the positive outcomes in the subset of patients with subchondral edema. Furthermore, drilling into the CBI is a mechanical stimulus for angiogenesis.

The economic advantage of the lower-cost PeCaBoo procedure is substantial when compared to the expenses of a total or partial knee replacement. It is performed as an outpatient, minimally invasive procedure that does not require an extended stay in the hospital post-procedure. The PeCaBoo procedure also has a lower risk of infection, blood clots, correction surgeries, and re-hospitalization than a knee replacement has. Together, these factors substantially reduce costs for both the patient and hospital.

Limitations of this study include the small number of participants and the subjectivity of self-reported outcomes. The NRS and WOMAC grading scales are based on patient-reported data, which is inherently subjective for each patient. To account for this, the MRI scans provide an unbiased comparison between patients. Future studies should expand the number of participants to increase the statistical power of the data.

\section{Conclusion}

In conclusion, PeCaBoo has the potential to be an alternative to total knee replacement when other minimally-invasive therapies have failed. Our study used mesenchymal stem cells from tibial bone marrow to treat knee osteoarthritis via PeCaBoo injection. To our knowledge, this is the first study to use this technique. Our results show that this procedure can improve pain and functioning scores, and also potentially repair or regenerate the intra-articular matrix at the chondral-bone interface. Early arthritis and presence of bone edema were positive prognostic factors in this pilot trial. As a cost-efficient and effective procedure to treat knee OA, our results from this PeCaBoo trial support the need for a study with a larger number of participants to confirm our findings.

\section{Acknowledgements}

We would like to thank Craig Sherman, M.D, for independently reading the MRI scans.

\section{References}

[1] Bhatia, D., Bejarano, T. and Novo, M. (2013) Current Interventions in the Management of Knee Osteoarthritis. Journal of Pharmacy and Bioallied Sciences, 5, 30-38. http://dx.doi.org/10.4103/0975-7406.106561

[2] Goldring, M.B. and Berenbaum, F. (2015) Emerging Targets in Osteoarthritis Therapy. Current Opinion in Pharmacology, 22, 51-63. http://dx.doi.org/10.1016/j.coph.2015.03.004 
[3] Naranje, S., Lendway, L., Mehle, S. and Gioe, T.J. (2015) Does Operative Time Affect Infection Rate in Primary Total Knee Arthroplasty? Clinical Orthopaedics and Related Research ${ }^{\circledR}$, 473, 64-69. http://dx.doi.org/10.1007/s11999-014-3628-4

[4] Brown, N.M., Sheth, N.P., Davis, K., et al. (2012) Total Knee Arthroplasty Has Higher Postoperative Morbidity than Unicompartmental Knee Arthroplasty: A Multicenter Analysis. The Journal of Arthroplasty, 27, 86-90. http://dx.doi.org/10.1016/j.arth.2012.03.022

[5] Farr, J., Tabet, S.K., Margerrison, E. and Cole, B.J. (2014) Clinical, Radiographic, and Histological Outcomes after Cartilage Repair with Particulated Juvenile Articular Cartilage: A 2-Year Prospective Study. American Journal of Sports Medicine, 42, 1417-1425. http://dx.doi.org/10.1177/0363546514528671

[6] Spahn, G., Klinger, H.M. and Hofmann, G.O. (2013) The Effect of Arthroscopic Debridement and Conservative Treatment in Knee Osteoarthritis Results of a 5-Year Follow-Up and Literature Review. Sportverletzung-Sportschaden, 27, 226-231.

[7] Findlay, D.M. (2007) Vascular Pathology and Osteoarthritis. Rheumatology (Oxford), 46, 1763-1768. http://dx.doi.org/10.1093/rheumatology/kem191

[8] Hong, Y.H., Park, C.W., Kim, H.S., Won, K.C., Kim, Y.W. and Lee, C.K. (2013) Effects of Hypoxia/Ischemia on Catabolic Mediators of Cartilage in a Human Chondrocyte, SW1353. Biochemical and Biophysical Research Communications, 431, 478-483. http://dx.doi.org/10.1016/j.bbrc.2013.01.035

[9] Sampson, S., Botto-van Bemden, A. and Aufiero, D. (2015) Regenerative Medicine: Stem Cell Therapies for Treatment of Cartilage and Bone Disorders: Osteoarthritis, Avascular Necrosis, and Non-Union Fractures. PM\&R, 7, S26-S32. http://dx.doi.org/10.1016/j.pmrj.2015.01.023

[10] Davies-Tuck, M., Kawasaki, R., Wluka, A.E., et al. (2012) The Relationship between Retinal Vessel Calibre and Knee Cartilage and BMLs. BMC Musculoskeletal Disorders, 13, 255-260. http://dx.doi.org/10.1186/1471-2474-13-255

[11] Matthews, G.L. and Hunter, D.J. (2011) Emerging Drugs for Osteoarthritis. Expert Opinion on Emerging Drugs, 16, 479-491. http://dx.doi.org/10.1517/14728214.2011.576670

[12] Davatchi, F., Abdollahi, B.S., Mohyeddin, M. and Nikbin, B. (2015) Mesenchymal Stem Cell Therapy for Knee Osteoarthritis: 5 Years Follow-Up of Three Patients. International Journal of Rheumatic Diseases, In Press. http://dx.doi.org/10.1111/1756-185X.12670

[13] Wong, K.L., Lee, K.B.L., Lee, E.H., Hui, J.H.P., Law, P. and Tai, B.C. (2013) Injectable Cultured Bone Marrow-Derived Mesenchymal Stem Cells in Varus Knees with Cartilage Defects Undergoing High Tibial Osteotomy: A Prospective, Randomized Controlled Clinical Trial with 2 Years' Follow-Up. Arthroscopy-Journal of Arthroscopic and Related Surgery, 29, 2020-2028.

[14] Sen, R.K., Tripathy, S.K., Aggarwal, S., Marwaha, N., Sharma, R.R. and Khandelwal, N. (2012) Early Results of Core Decompression and Autologous Bone Marrow Mononuclear Cells Instillation in Femoral Head Osteonecrosis. A Randomized Control Study. Journal of Arthroplasty, 27, 679-686. http://dx.doi.org/10.1016/j.arth.2011.08.008

[15] Narbona-Carceles, J., Vaquero, J., Susana Suárez-Sancho, B.S., Forriol, F. and Fernández-Santos, M.E. (2014) Bone Marrow Mesenchymal Stem Cell Aspirates from Alternative Sources Is the Knee as Good as the Iliac Crest? Injury, 45, S42-S47. http://dx.doi.org/10.1016/S0020-1383(14)70009-9

[16] Smiler, D.G., Soltan, M., Soltan, C. and Matthews, C. (2010) Growth Factors and Gene Expression of Stem Cells: Bone Marrow Compared with Peripheral Blood. Implant Dentistry, 19, 229-240. http://dx.doi.org/10.1097/ID.0b013e3181dc24a9

[17] Fiedler, J., Röderer, G., Günther, K.P. and Brenner, R.E. (2002) BMP-2, BMP-4, and PDGF-bb Stimulate Chemotactic Migration of Primary Human Mesenchymal Progenitor Cells. Journal of Cellular Biochemistry, 87, 305-312. http://dx.doi.org/10.1002/jcb.10309

[18] Sato, N., Beitz, J.G., Kato, J., et al. (1993) Platelet-Derived Growth Factor Indirectly Stimulates Angiogenesis in Vitro. American Journal of Pathology, 142, 1119-1130.

[19] Ferrara, N. (1999) Role of Vascular Endothelial Growth Factor in the Regulation of Angiogenesis. Kidney International, 56, 794-814. http://dx.doi.org/10.1046/j.1523-1755.1999.00610.x

[20] Ribatti, D. (2005) The Crucial Role of Vascular Permeability Factor/Vascular Endothelial Growth Factor in Angiogenesis: A Historical Review. British Journal of Haematology, 128, 303-309. http://dx.doi.org/10.1111/j.1365-2141.2004.05291.x

[21] Ishibashi, H., Nariai, Y., Kanno, T., Onimaru, M. and Sekine, J. (2014) Effects of Transforming Growth Factor Beta 1 on the Plasminogen Activation System, Collagen and Integrin Synthesis, and Proliferation of Rabbit Mandibular Condylar Chondrocytes. International Journal of Oral \& Maxillofacial Surgery, 43, 470-475. http://dx.doi.org/10.1016/j.ijom.2013.10.010

[22] Joyce, M.E., Roberts, A.B., Sporn, M.B. and Bolander, M.E. (1990) Transforming Growth Factor-Beta and the Initia- 
tion of Chondrogenesis and Osteogenesis in the Rat Femur. Journal of Cell Biology, 110, 2195-2207. http://dx.doi.org/10.1083/jcb.110.6.2195

[23] De Luca, F., Barnes, K.M., Uyeda, J.A., et al. (2001) Regulation of Growth Plate Chondrogenesis by Bone Morphogenetic Protein-2. Endocrinology, 142, 430-436. http://dx.doi.org/10.1210/en.142.1.430

[24] Charalambous, C.P. (2014) Cell Origin and Differentiation in the Repair of Full-Thickness Defects of Articular Cartilage. In: Banaszkiewicz, P.A. and Kader, D.F., Eds., Classic Papers in Orthopaedics, Springer, London, 377-379. http://dx.doi.org/10.1007/978-1-4471-5451-8_95

[25] Chevrier, A., Hoemann, C.D., Sun, J. and Buschmann, M.D. (2007) Chitosan-Glycerol Phosphate/Blood Implants Increase Cell Recruitment, Transient Vascularization and Subchondral Bone Remodeling in Drilled Cartilage Defects. Osteoarthritis and Cartilage, 15, 316-327. http://dx.doi.org/10.1016/j.joca.2006.08.007

[26] Gomoll, A.H., Madry, H., van Dijk, N., et al. (2010) The Subchondral Bone in Articular Cartilage Repair: Current Problems in the Surgical Management. Knee Surgery, Sports Traumatology, Arthroscopy, 18, 434-447. http://dx.doi.org/10.1007/s00167-010-1072-x

[27] “Pain Intensity Instruments”. National Institutes of Health—Warren Grant Magnuson Clinical Center. July 2003. Archived from the Original (PDF) on 9 November 2015.

[28] American College of Rheumatology (2015) Western Ontario and McMaster Universities Osteoarthritis Index (WOMAC)-General Description. ACR.

[29] Miletic, K.G., Taylor, T.N., Martin, E.T., Vaidya, R. and Kaye, K.S. (2014) Readmissions after Diagnosis of Surgical Site Infection Following Knee and Hip Arthroplasty. Infection Control \& Hospital Epidemiology, 35, 152-157. http://dx.doi.org/10.1086/674854

[30] van der Weegen, W., Wullems, J.A., Bos, E., Noten, H. and van Drumpt, R.A. (2015) No Difference between IntraArticular Injection of Hyaluronic Acid and Placebo for Mild to Moderate Knee Osteoarthritis: A Randomized, Controlled, Double-Blind Trial. Journal of Arthroplasty, 30, 754-757. http://dx.doi.org/10.1016/j.arth.2014.12.012

[31] Bannuru, R.R., Vaysbrot, E.E., Sullivan, M.C. and McAlindon, T.E. (2014) Relative Efficacy of Hyaluronic Acid in Comparison with NSAIDs for Knee Osteoarthritis: A Systematic Review and Meta-Analysis. Seminars in Arthritis and Rheumatism, 43, 593-599. http://dx.doi.org/10.1016/j.semarthrit.2013.10.002

[32] Ayhan, E., Kesmezacar, H. and Akgun, I. (2014) Intra-Articular Injections (Corticosteroid, Hyaluronic Acid, Platelet Rich Plasma) for the Knee Osteoarthritis. World Journal of Orthopedics, 5, 351-361. http://dx.doi.org/10.5312/wjo.v5.i3.351

[33] Patrício, J.P., Barbosa, J.P., Ramos, R.M., Antunes, N.F. and de Melo, P.C. (2013) Relative Cardiovascular and Gastrointestinal Safety of Non-Selective Non-Steroidal Anti-Inflammatory Drugs versus Cyclo-Oxygenase-2 Inhibitors: Implications for Clinical Practice. Clinical Drug Investigation, 33, 167-183. http://dx.doi.org/10.1007/s40261-013-0052-6

[34] Trelle, S., Reichenbach, S., Wandel, S., et al. (2011) Cardiovascular Safety of Non-Steroidal Anti-Inflammatory Drugs: Network Meta-Analysis. BMJ: British Medical Journal, 342, c7086.

[35] Kazakia, G.J., Kuo, D., Schooler, J., et al. (2013) Bone and Cartilage Demonstrate Changes Localized to Bone Marrow Edema-Like Lesions within Osteoarthritic Knees. Osteoarthritis and Cartilage, 21, 94-101. http://dx.doi.org/10.1016/j.joca.2012.09.008

[36] Wallace, G., Judge, A., Prieto-Alhambra, D., de Vries, F., Arden, N.K. and Cooper, C. (2014) The Effect of Body Mass Index on the Risk of Post-Operative Complications during the 6 Months Following Total Hip Replacement or Total Knee Replacement Surgery. Osteoarthritis and Cartilage, 22, 918-927. http://dx.doi.org/10.1016/j.joca.2014.04.013

[37] Garvin, K.L. and Konigsberg, B.S. (2012) Infection Following Total Knee Arthroplasty: Prevention and Management. Instructional Course Lectures, 61, 411-419.

[38] Murphy, L. and Helmick, C.G. (2012) The Impact of Osteoarthritis in the United States: A Population-Health Perspective: A Population-Based Review of the Fourth Most Common Cause of Hospitalization in US Adults. American Journal of Nursing, 112, S13-S19. http://dx.doi.org/10.1097/01.NAJ.0000412646.80054.21

[39] Whitehouse, J.D., Friedman, N.D., Kirkland, K.B., Richardson, W.J. and Sexton, D.J. (2002) The Impact of Surgical-Site Infections Following Orthopedic Surgery at a Community Hospital and a University Hospital: Adverse Quality of Life, Excess Length of Stay, and Extra Cost. Infection Control and Hospital Epidemiology, 23, 183-189. http://dx.doi.org/10.1086/502033

[40] Waimann, C.A., Fernandez-Mazarambroz, R., Cantor, S.B., et al. (2014) Cost-Effectiveness of Total Knee Replacement: A Prospective Cohort Study. Arthritis Care \& Research, 66, 592-599. http://dx.doi.org/10.1002/acr.22186

[41] Aigner, T., Söder, S., Gebhard, P.M., Mc Alinden, A. and Haag, J. (2007) Mechanisms of Disease: Role of Chondrocytes in the Pathogenesis of Osteoarthritis-Structure, Chaos and Senescence. Nature Clinical Practice Rheumatology, 
3, 391-399. http://dx.doi.org/10.1038/ncprheum0534

[42] Roman-Blas, J.A. and Jimenez, S.A. (2006) NF- $\kappa$ B as a Potential Therapeutic Target in Osteoarthritis and Rheumatoid Arthritis. Osteoarthritis and Cartilage, 14, 839-848. http://dx.doi.org/10.1016/j.joca.2006.04.008

[43] Noth, U., Steinert, A.F. and Tuan, R.S. (2008) Technology Insight: Adult Mesenchymal Stem Cells for Osteoarthritis Therapy. Nature Clinical Practice Rheumatology, 4, 371-380. http://dx.doi.org/10.1038/ncprheum0816

[44] Caplan, A.I. and Dennis, J.E. (2006) Mesenchymal Stem Cells as Trophic Mediators. Journal of Cellular Biochemistry, 98, 1076-1084. http://dx.doi.org/10.1002/jcb.20886

[45] Pittenger, M.F., Mackay, A.M., Beck, S.C., et al. (1999) Multilineage Potential of Adult Human Mesenchymal Stem Cells. Science, 284, 143-147. http://dx.doi.org/10.1126/science.284.5411.143

[46] Beane, O.S., Darling, E.M., Fonseca, V.C., Cooper, L.L. and Koren, G. (2014) Impact of Aging on the Regenerative Properties of Bone Marrow-, Muscle-, and Adipose-Derived Mesenchymal Stem/Stromal Cells. PLoS ONE, 9, e115963. http://dx.doi.org/10.1371/journal.pone.0115963

[47] Bouquot, J.E., McMahon, R.E. and Glueck, C.J. (2008) Bone Marrow Edema: Mild or Nascent Variant of Ischemic Bone Disease. Journal of Oral and Maxillofacial Surgery, 66, 205-208. http://dx.doi.org/10.1016/j.joms.2007.08.017

[48] Grellier, M., Bordenave, L. and Amédée, J. (2009) Cell-to-Cell Communication between Osteogenic and Endothelial Lineages: Implications for Tissue Engineering. Trends in Biotechnology, 27, 562-571. http://dx.doi.org/10.1016/j.tibtech.2009.07.001 